msh-mss Mathématiques et sciences humaines

188 | Hiver 2009

Varia

\title{
Évolution log-périodique des ordres religieux et monastiques
}

Log periodic evolultion of monastic orders

\section{Ivan Brissaud}

\section{(2) OpenEdition}

12 Journals

Édition électronique

URL : http://journals.openedition.org/msh/11234

DOI : $10.4000 / \mathrm{msh} .11234$

ISSN : 1950-6821

Éditeur

Centre d'analyse et de mathématique sociales de l'EHESS

\section{Édition imprimée}

Date de publication : 31 décembre 2009

Pagination : 55-65

ISSN : 0987-6936

\section{Référence électronique}

Ivan Brissaud, «Évolution log-périodique des ordres religieux et monastiques », Mathématiques et sciences humaines [En ligne], 188 | Hiver 2009, mis en ligne le 15 mars 2010, consulté le 23 juillet 2020 URL : http://journals.openedition.org/msh/11234; DOI : https://doi.org/10.4000/msh.11234

\section{(c) École des hautes études en sciences sociales}




\title{
ÉVOLUTION LOG-PÉRIODIQUE DES ORDRES RELIGIEUX ET MONASTIQUES
}

\author{
Ivan BRISSAUD ${ }^{1}$
}

\begin{abstract}
RÉSUMÉ - À partir du VIe siècle les ordres religieux et monastiques majeurs se sont succédés en marquant les époques par leurs réalisations et leurs influences dans le domaine social, économique et politique. L'un après l'autre ils ont essayé de maintenir ou de faire renaître une observance plus ou moins stricte des "règles " fondatrices. Mais à chaque tentative ces buts ne purent être atteints; aussi chaque ordre dominant finissait par être supplanté par la fondation d'un nouvel ordre. Le but de cette analyse est de vérifier si cette succession s'inscrit dans un modèle log-périodique comme cela a été montré pour d'autres chronologies. La conclusion est que, dans ce modèle, le temps critique vers lequel tend cette évolution se situe au début du XIVe siècle et qu'après un long immobilisme les ordres "réguliers 》 ont perdu leur hégémonie au profit des religieux séculiers.
\end{abstract}

MOTS-CLÉS - Arbre d'évolution, Log-Périodicité, Ordres religieux

SUMMARY - Log periodic evolution of monastic orders

After the VIth century various religious and monastic orders, all of which influenced the social, economical and political life of their time, succeeded each other. These orders all attempted to promote a stricter respect of the fundamental (religious) rules. But failing to achieve this goal, each order was 7 supplanted by a new one. The succession of jumps in this evolutionary tree shows an acceleration between Benedictine up to Mendicant orders (XIIIth century) similar to what has been observed for various phenomena (evolution of hominidae, financial market crash, earthquake....). This suggests that it might be relevant to apply a log periodic system to this chronology. The deduced values of the critical times and of the self similarity factor are $T c=1290+/-40$ and $g=2,14+/-0,30$. No deceleration steps are observed after Tc. This could be explained by the predominance of the secular clergy.

KEYWORDS - Log-Periodicity, Monastic orders, Temporal tree structure

\section{INTRODUCTION}

D. Sornette, A. Johansen et J.-P. Bouchaud [1996] ont analysé avec des lois logpériodiques l'évolution des indices économiques $S$ et $P 500$ avant que A. Johansen et D. Sornette [1999] n'appliquent des lois de même nature à l'évolution des indices Nikkei et Dow Jones en montrant la possibilité d'une prévision des krachs financiers. D. Sornette [1998] développait alors la notion d'invariance d'échelle discrète ayant des dimensions complexes. Simultanément, de son coté, L. Nottale proposait la relativité d'échelles [1998] et dans ce cadre, par une série d'articles, il étudiait avec J. Chaline et P. Grou [1999] les évolutions de diverses espèces (hominidés, équidés, rongeurs...) ainsi que la chronologie des crises économiques depuis 10000 ans dans le monde

\footnotetext{
${ }^{1}$ Directeur de recherche honoraire IN2P3/ CNRS, Université Paris-Sud, brissaud.ivan@orange.fr
} 
I. BRISSAUD

occidental et l'Amérique Centrale. Pour notre part, plus modestement, nous avons voulu mettre en évidence deux autres log-périodicités dans des domaines et des durées différentes : celle de la musique Jazz [Brissaud, 2007] et, en collaboration [Brissaud, Baron, 2007], celle des accélérateurs de particules utilisés en physique nucléaire et corpusculaire.

Dans le présent travail nous souhaitons montrer que les ordres religieux et monastiques majeurs évoluent également selon une loi log-périodique. Dans un article précédent [Brissaud, 2004] nous avons déjà proposé d'expliquer par des systèmes critiques auto-organisés la croissance de ces ordres entre le Ve et XVIe siècles.

\section{LOI LOG-PÉRIODIQUE}

La log- périodicité s'applique à des systèmes dont l'évolution est, certes, régulière mais discontinue car coupée par des sauts notables qui donnent naissance à des évolutions secondaires. On peut faire l'analogie avec le point de départ des branches pour les arbres végétaux. On trouvera le formalisme et ses justifications dans les articles de L. Nottale, J. Chaline et P. Grou cités plus haut.

Les sauts (les discontinuités ou embranchements) au cours d'une évolution se succèdent à des instants Tn tels que

$$
\log (\mathrm{Tn}-\mathrm{Tc})=\log \left(\mathrm{T}^{\circ}-\mathrm{Tc}\right)-\mathrm{n} \times \log (\mathrm{g})
$$

où $\mathrm{Tc}$ est le moment qui correspond à la limite finale théorique de l'évolution après $\mathrm{n}$ sauts aux temps $\mathrm{Tn}$. $\mathrm{T}^{\circ}$ est une constante et $\mathrm{g}$ le facteur d'auto similarité déterminant les séries d'intervalles de temps en échelles logarithmiques $\log (\mathrm{Tn}-\mathrm{Tc})$. Les deux inconnues Tc et $g$ peuvent être déterminées à partir de deux dates évènementielles successives :

$$
\begin{gathered}
\mathrm{g}=(\mathrm{Tn}-\mathrm{Tn}-1) /(\mathrm{Tn}+1-\mathrm{Tn})=(\mathrm{Tn}-\mathrm{Tc}) /(\mathrm{Tn}+1-\mathrm{Tc}) \\
\mathrm{Tc}=(\mathrm{g} \times \mathrm{Tn}+1-\mathrm{Tn}) /(\mathrm{g}-1)
\end{gathered}
$$

Ainsi, connaissant les paramètres Tc et g, il est aisé de calculer l'instant $\mathrm{Tn}+1 \mathrm{de}$ l'événement de rang $\mathrm{n}+1$ à partir de l'instant Tn de rang $\mathrm{n}$ :

$$
\mathrm{Tn}+1=[(\mathrm{g}-1) \times \mathrm{Tc}+\mathrm{Tn}] / \mathrm{g}
$$

et ainsi de suite. Ceci suppose a priori que l'arbre généalogique est entièrement logpériodique et avec la même auto-similarité.

Une conclusion notable de ce simple modèle est, s'il est vérifié, d'affirmer qu'il est possible de prédire approximativement les dates des étapes d'une chronologie dont on connaît les paramètres $\mathrm{g}$ et $\mathrm{Tc}$, mais seulement les dates et non leurs causes et leurs conséquences. La principale question posée est de comprendre l'origine d'une telle loi d'arborescence fractale.

\section{CHRONOLOGIE SUCCINTE DES ORDRES DU VIe AU XXe SIÈCLE}

Pour comprendre la raison de l'application de la log-périodicité aux ordres religieux et monastiques, survolons l'histoire de ceux-ci et leurs chronologies. Les éléments de ce résumé sont extraits, en grande partie, des références [Marc-Bonnet, 1968 ; Le Bras, 1980 ; Le Goff, 1988 ; Berlioz \& al., 1994 ; Dubois, 1998]. Il ne s'agit évidemment pas 
d'un exercice d'historien, mais de mettre en évidence l'évolution globale, « statistique », des ordres majeurs.

Dés les premiers siècles, des chrétiens se rassemblent pour méditer, étudier la Bible (le monachisme) alors que d'autres (les ermites) poursuivent les mêmes buts dans la solitude, souvent dans les déserts égyptiens. Le monachisme a diffusé depuis l'Est méditerranéen vers l'Occident, spécialement par la Provence (Iles de Lérins). Rappelons simplement les noms de saint Jérôme, saint Augustin qui ont eu une forte influence au IVe siècle. Une étape considérable est franchie quand saint Benoît crée vers 530 le monastère italien du Mont Cassin et commence à rédiger la « règle bénédictine » qui organise la pratique religieuse et le mode de vie de l'ensemble des moines vivant dans les établissements religieux. Si d'autres règles sont proposées, c'est la "bénédictine » qui reste la plus suivie. À partir de cette époque le monachisme se développe fortement dans tout le monde occidental. Le nombre d'établissements bénédictins dépasse largement 2500. Mais, au VIIIe siècle carolingien, des difficultés se manifestent, en particulier au sujet du rapport des monastères avec le pouvoir politique royal et féodal (question toujours présente jusqu'à nos jours). Au IXe siècle le système bénédictin est fortement ébranlé par ces problèmes politiques, mais aussi par les guerres, invasions et pillages qui ravagent les biens des moines. Ceci conduit à la destruction de nombreux établissements et à des regroupements vers des lieux plus sûrs.

C'est au monastère bénédictin de Cluny, fondé en 910, que revient la charge de réactiver la règle de saint Benoît. Si cette réforme prend naissance à Cluny, elle s'étend rapidement à d'autres abbayes qui se placent sous sa tutelle. Un atout important de ce monastère est d'avoir eu des abbés issus de grandes familles seigneuriales, ce qui favorise l'entente avec cette classe sociale dont le poids est primordial à cette époque alors que le pouvoir royal devient déficient. À cela s'ajoute « l'exemption » accordée par le pape, décision qui coupe tout lien de dépendance vis-à-vis de l'évêque local, et qui relie le destinataire uniquement au Vatican. Ainsi, les abbés successifs de Cluny bénéficient de ce privilège pour eux-mêmes ; également les abbés des dépendances (celles-ci dépassent 1100) l'obtiennent. Sur un plan plus matériel, cette décision supprime la dîme due aux évêques. Ainsi l'ordre clunisien est en phase avec l'organisation sociale du moment. Sa richesse, sa puissance eurent un effet considérable sur les différentes sociétés occidentales qui étaient en pleine réorganisation. Cependant au début du XIIe siècle des excès, une puissance trop grande créent des résistances qu'accompagnent un relâchement, même une sclérose. C'est le déclin.

À la même époque est fondé le monastère de Cîteaux par Robert de Molesme. Son but et celui des moines qui l'accompagnent est de revenir à l'observance stricte bénédictine (1098). Quelques années plus tard Cîteaux s'est déjà fortement développé quand saint Bernard quitte l'établissement pour prendre la direction de l'abbaye de Clairvaux (1112). De là il va accroître considérablement l'influence de l'ordre et le nombre de « filles » de l'abbaye-mère, c'est-à-dire de dépendances (550 monastères vers 1250 et environ 700 à la fin du siècle). Selon une recette classique, la croissance de Cîteaux s'effectue de deux façons : fondation de nouveaux établissements ou accueil d'anciens établissements d'un vieil ordre (ces changements de camp se font également en sens inverse). La prospérité est obtenue par une recherche spirituelle très exigeante, un travail acharné, mais aussi grâce au soutien de la papauté car Bernard et beaucoup d'autres cisterciens vont sortir de leurs établissements pour servir avec efficacité les intérêts des papes dans des problèmes séculiers (appel à la seconde croisade, intervention pour les élections papales, critiques d'évêques...). L'exemption dont bénéficie également l'ordre sert de liant entre les abbayes. Les cisterciens ont beaucoup 
de succès dans de nombreux domaines, mais plus spécialement dans l'exploitation de leurs nombreuses et vastes possessions. Ceci les pousse vers de multiples activités économiques d'où un très grand enrichissement conduisant à des contradictions avec leurs principes premiers ; toutes ces raisons mènent à une certaine décadence.

Selon le schéma classique, des tentatives de réforme ont lieu dans diverses directions. Parmi elles, deux sont de grands succès : les fondations quasi simultanées vers 1209 des ordres dominicains (ou prêcheurs) et franciscains (ou mineurs) qui constituent les ordres mendiants (ainsi nommés car ils vivent de dons et non de la dîme). Les Dominicains suivent la règle de saint Augustin et les Franciscains celle de saint François d'Assise. C'est le moment de la création et du développement des villes et aussi de la diffusion des hérésies. En conséquence les « Mendiants » vont s'implanter dans les cités de préférence aux monastères de campagne pour, principalement, lutter contre toutes les déviations religieuses. Par opposition à l'opulence des couvents cisterciens, ils prêchent la pauvreté.

Enfin, vers 1256, les ermites dits de « saint Augustin » rejoignent les Mendiants, précédés de peu par les Carmes (des ermites à l'origine). Ces nouveaux venus ont été protégés en grande partie du relâchement par leur érémitisme qui les a maintenus dans un mode de vie très strict. C'est pour renforcer les Mendiants que le pape décide de les inclure parmi ceux-ci. Assez rapidement le développement de ces ordres, leur indépendance, leurs implantations dans les agglomérations et l'impact de leurs prêches dans les églises et les lieux publics amènent des difficultés de cohabitation avec le clergé séculier. Les abus des «commanditaires », personnes non religieuses à qui la hiérarchie donne la direction d'un établissement et qui l'exploitent souvent à leur propre profit, créent un état d'esprit déplorable. Simultanément des oppositions internes apparaissent à propos de la question de la pauvreté des ordres. Donc, en moins d'un siècle, des clivages, des troubles violents affaiblissent tous les ordres mendiants. La dernière fondation a lieu en 1256 et la suivante en 1435 seulement !

Terminons ces rappels concernant les ordres majeurs par deux remarques :

- L'ordre des Chartreux se développa lentement à partir du XIIIe siècle, mais nous le mettons à part du fait de sa spécificité érémitique qui l'isole du monde.

- D'autres ordres apparaissent à la même époque, mais ils ont eu un rôle et une influence bien moins marquants. Ils constituent des tentatives de réformes, des essais d'innovations qui restent limités, peut-être par absence d'un leader charismatique ou d'un projet clair.

Durant les trois-quarts du XIVe siècle les ordres subissent une stagnation très marquée, même une certaine décadence. Des tentatives de réforme sont faites sans grand succès. Beaucoup d'établissements s'isolent, refusent tout retour à la Règle, végètent et souvent disparaissent. À la fin du siècle un léger renouveau lié à la meilleure santé économique se manifeste. L'évolution des ordres est alors fort différente de celle qui a marqué le pas vers l'an 1300 lorsqu'il n'y avait que quelques ordres notables, très charpentés et homogènes qui se succédaient pour maintenir, sinon améliorer une influence religieuse sur les sociétés occidentales qu'ils voulaient réformer, se considérant comme les soldats de Dieu. À partir du XIVe siècle les conflits internes, en particulier entre partisans de la stricte observance et les autres, persistent ou s'intensifient jusqu'à conduire à des excommunications. Les papes ne pouvant ou ne voulant pas imposer une seule observance se résignent à autoriser et régenter des scissions : stricte et commune observances. Face à cette division, des regroupements sont nécessaires ; ceux-ci se font parfois dans une même région, mais souvent, au gré 
des affinités religieuses, dans des pays différents. Vers l'an 1500 il y a un début de réforme reliée à la fondation de congrégations et à la division des ordres. Ainsi la régénération des Franciscains correspond à l'arrivée des Récollets et des Capucins ; les Carmes se séparent en Carmes Déchaux et Chaussés. Les Cisterciens se divisent en congrégations (les Feuillants...) alors qu'est fondé l'ordre des Trappistes. Les congrégations sont nombreuses chez les Clunisiens (saint Vanne et saint Maur) et chez les Dominicains (saint Louis). Mais, au Siècle des Lumières, à partir de sa deuxième moitié, les ordres sont attaqués par les pouvoirs laïques. Les Jésuites sont interdits vers 1760. En France, après la Révolution, les biens ecclésiastiques sont saisis, vendus et souvent pillés et détruits. Au cours de la période napoléonienne en Europe les ordres sont dispersés ou interdits, très proches de la disparition totale. Si après 1820 il y a une renaissance notable grâce à des religieux ayant survécu aux épreuves, les conflits avec les pouvoirs publics redoublent dès la fin du XIXe siècle. Symbole de cette décadence : des abbayes naguère prestigieuses, des lieux de méditation comme Clairvaux, deviennent des prisons, certes pour condamnés politiques ! Un certain statu quo s'établit après la première guerre mondiale.

Tout ceci explique un large éparpillement d'autant plus que, simultanément, un grand nombre d'ordres nouveaux sont crées ; mais ils sont d'une envergure médiocre . Il en résulte une sorte d'atomisation des établissements, avec un grand nombre de nuances dans les modes de fonctionnement. La Figure 1 montre les deux phases décrites plus haut : la succession des ordres jusque vers 1300, suivie des divisions avant des regroupements aux XVIe et XIXe siècles.

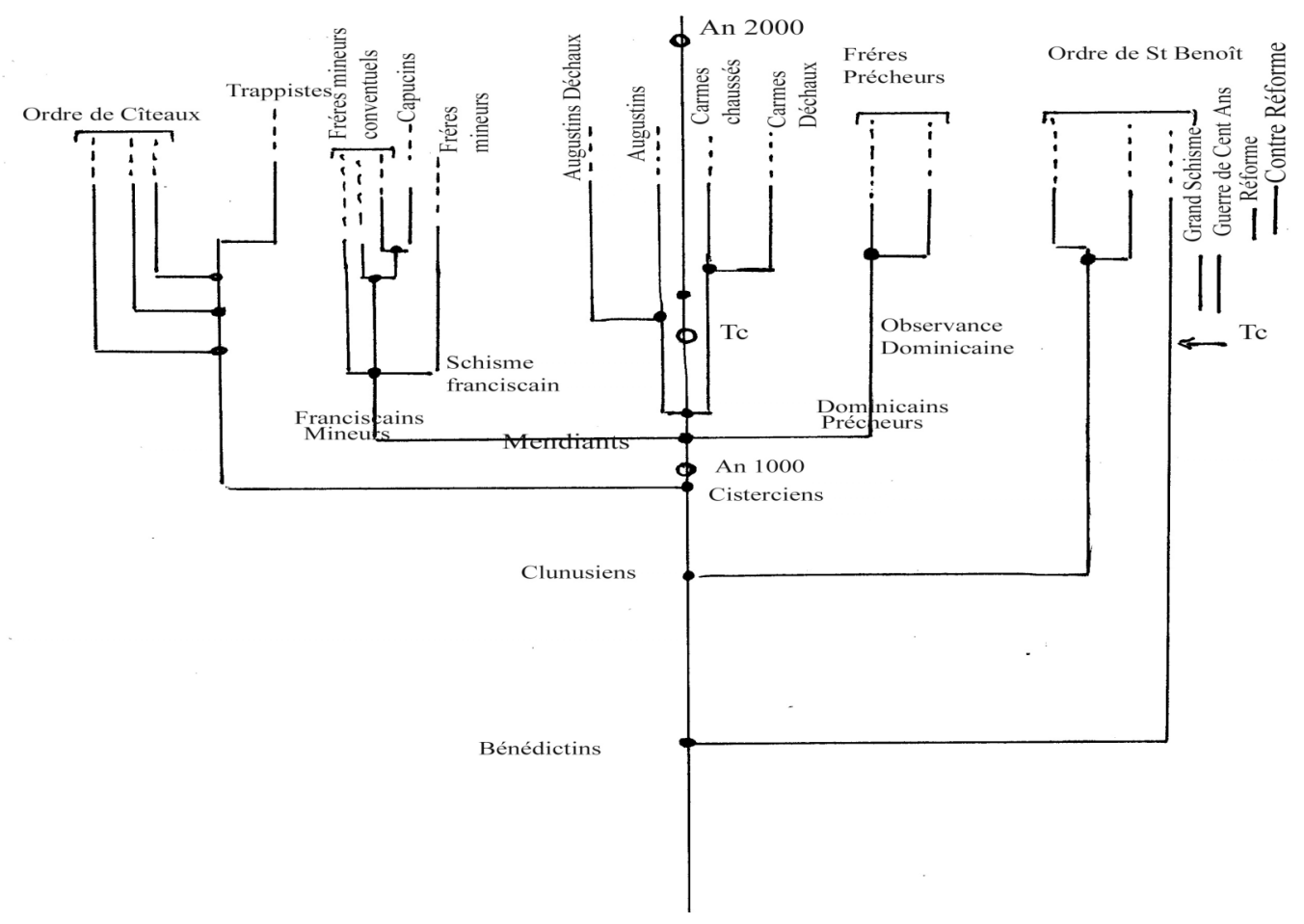

FIGURE 1. Arbre généalogique simplifié des ordres majeurs jusqu'aux regroupements du XVIIe et XIXe siècles

Sur la Figure 2, la courbe semi-logarithmique donne, en fonction de leurs dates de fondation, le nombre cumulé d'ordres d'une certaine importance subsistant actuellement. Rappelons que dans une telle représentation une fonction exponentielle apparaît comme 
une droite. Donc, cette figure confirme que la croissance de ce nombre d'ordres, au cours des deux derniers siècles, est super exponentielle prouvant que l'on s'approche de la limite de créations d'ordres alors que le recrutement de religieux diminue lentement. Cette conclusion est consistante avec l'historique précédent. Pour prolonger cette étude il serait intéressant de disposer du nombre de religieux et d'établissements pour l'ensemble des ordres afin d'apprécier leurs variations entre le XIVe et le XXe siècles. Mais, malheureusement, de telles statistiques n'existent pas actuellement.

N Nombre cumulé d'ordres

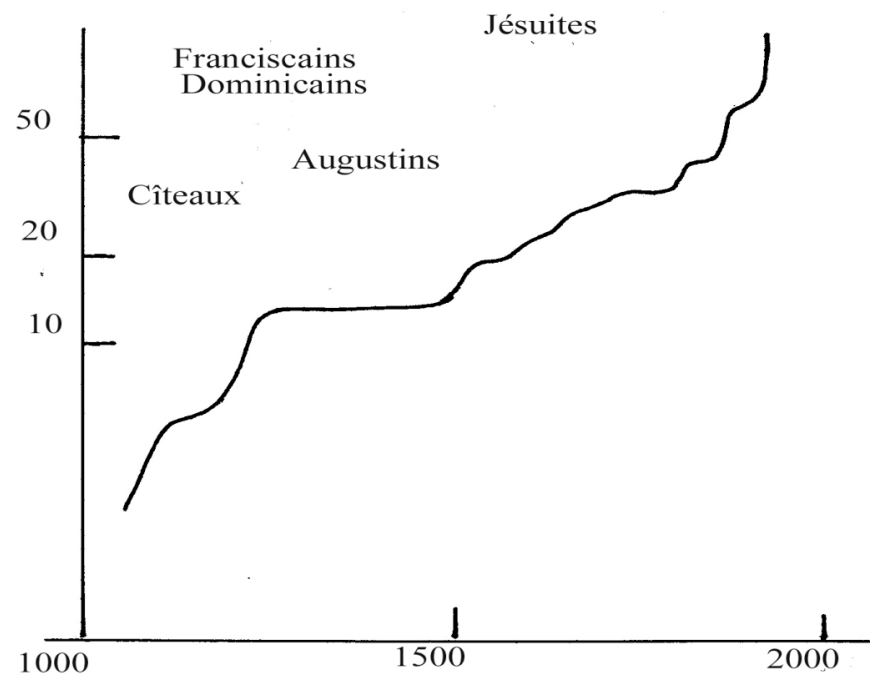

FIGURE 2. Nombre cumulé des ordres fondés depuis l'an 1000

\section{DISCUSSION}

Rappelons quelques résultats de l'article référencé [Brissaud, 2004] qui avait pour but de vérifier des hypothèses sur la naissance de chacun des principaux ordres (système critique auto-organisé) et non d'étudier leurs successions :

Le nombre de dépendances que possèdent les ordres évoqués plus haut croît avec le temps en suivant parfaitement une fonction logistique de la forme

$$
\mathrm{N}=\mathrm{N}^{\circ} / 1+\exp -\left(\left(\mathrm{t}-\mathrm{t}^{\circ}\right) / \mathrm{Dt}\right)
$$

où Dt est un paramètre lié à la durée de la croissance. Pour chacun des ordres la valeur de Dt est différente, mais la courbe donnant le logarithme de Dt en fonction de son classement est une droite, ce qui signe la présence d'une loi puissance. La Figure 3, à titre d'exemple, donne le nombre de monastères franciscains en fonction du temps (cf. Figure 3a) ainsi que la décomposition en deux logistiques obtenue grâce au code Loglet (cf. Figure 3b). Les caractéristiques de ces logistiques sont visibles sur les tableaux des figures. 


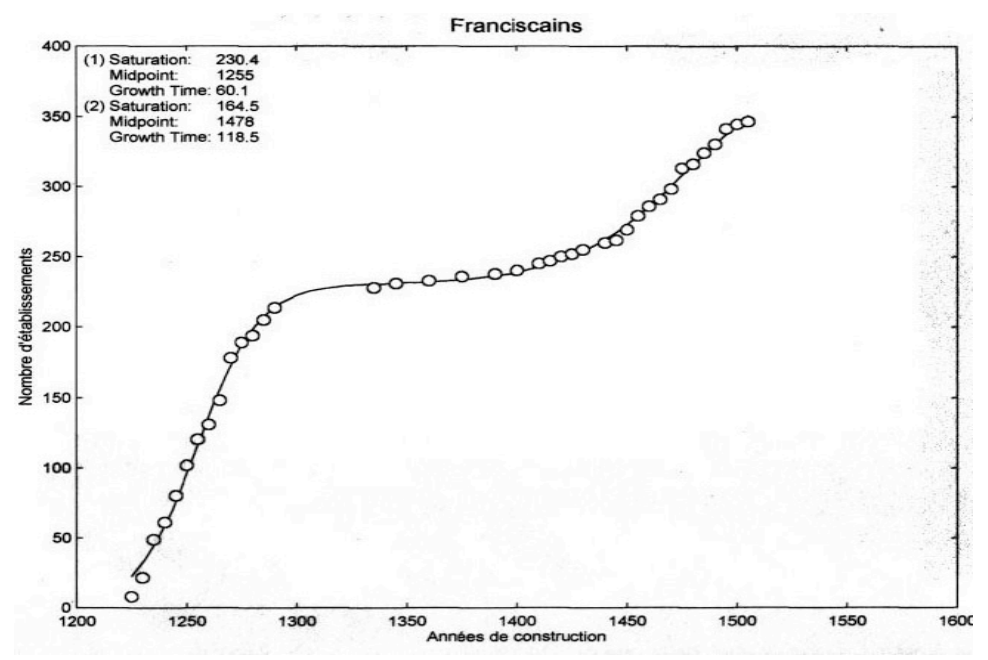

FIGURE 3a

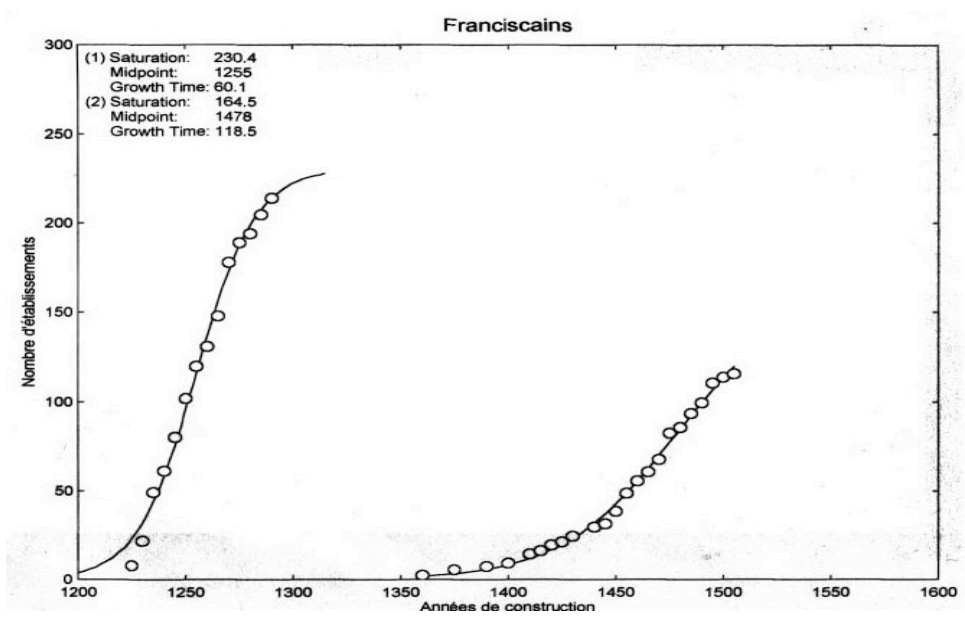

FIGURE $3 b$

Les distributions géographiques en Europe occidentale des établissements appartenant respectivement aux ordres clunisien et cistercien sont fractales. Nous l'avons établi pour ces deux seuls ordres car la localisation de leurs monastères (très nombreux) est bien connue (cf. Figure 4). Cette conclusion résulte d'une analyse radiale, centrée respectivement sur Cluny et Cîteaux, selon la technique développée par P. Frankhauser [1994]. Le rayon de ségrégation, limite de la zone fractale, est environ $800 \mathrm{~km}$ et $1100 \mathrm{~km}$ et les dimensions fractales sont respectivement $1,32+/-0,05$ et $1,52+/-0,03$. D'autre part, si on établit la courbe donnant le nombre total de monastères de chacun des ordres en fonction de son classement, on constate également une loi puissance.

Chaque ordre subit une certaine décadence, mais il est incapable de se réformer de lui-même. Les causes du déclin sont, certes, toujours internes, mais aussi externes dans des conflits avec les autorités royales, seigneuriales, papales, épiscopales et plus généralement avec le clergé séculier. D'autre part, il faut constater que c'est toujours à partir d'un site différent que démarre le processus de réforme ; comme l'a écrit P. Grou [1987], il y a « des changements de lieux de domination ». 


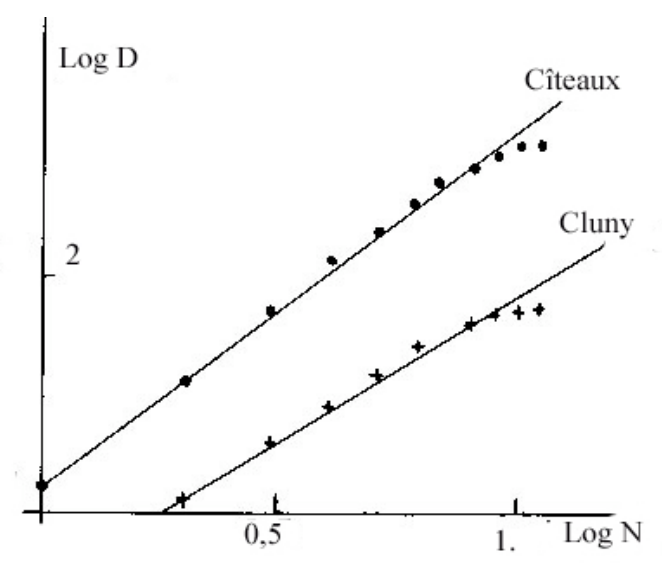

FIGURE 4. Nombres de dépendances de Cluny et Cîteaux en fonction de leurs distances $\left(\mathrm{N}^{*} 140 \mathrm{~km}\right)$ à ces deux centres.

Les échelles sont logarithmiques

Il est montré dans l'article [Brissaud, 2004] qu'il y a une hiérarchisation à l'intérieur de chacun des ordres. Le développement de ceux-ci s'effectue à partir d'un centre (Cluny, Cîteaux...) avec diffusion d'établissement en établissement. Ainsi s'établit une dépendance entre abbaye-mère et abbaye-fille, etc... La hiérarchie entre les ordres est moins marquée ; elle se traduit dans les faits quand le pape autorise un nouvel ordre, favorise son développement (exemptions par exemple) et surtout lui confie de lourdes responsabilités (inquisition notamment).

À partir de l'historique précédent on peut faire apparaître un arbre généalogique dans la succession des ordres majeurs examinés plus haut. Ils se sont succédé durant plus de sept siècles après avoir dominé le monde chrétien sur des plages de temps de plus en plus réduites de quatre siècles environ à moins de un. Le processus reste identique : les ordres veulent revenir à la pureté primitive, aux principes initiaux, à de nouvelles pratiques pour toujours mieux servir leur Dieu et son Église ; mais après une période faste ils retombent dans un certain déclin plus ou moins net. On peut en conclure qu'ils ont atteint leur limite de créativité. Il faut, alors, qu'un nouvel ordre émerge et devienne prépondérant à son tour. L. Nottale, J. Chaline et P. Grou [en particulier Nottale, 2000] ont bien mis en évidence de telles séquences et accélérations dans les exemples qu'ils ont analysés. Nous retrouvons donc tous les arguments pour proposer qu'une loi log-périodique soit applicable au cas présent.

Rappelons les dates approximatives des fondations des ordres étudiés ici :

\begin{tabular}{|l|c|}
\hline \multicolumn{1}{|c|}{ Ordres } & Année de fondation \\
\hline Bénédictins & 530 \\
Cluny & 910 \\
Cîteaux & 1098 \\
Franciscains & 1209 \\
Dominicains & 1209 \\
Carmes & 1256 \\
Augustins & 1256 \\
\hline
\end{tabular}


L'ajustement du modèle log-périodique permet de calculer les deux paramètres intervenant dans les formules I. Le résultat du calcul est :

$$
\begin{gathered}
\mathrm{g}=2,14+/-0,30 \\
\mathrm{Tc}=1290+/-40
\end{gathered}
$$

Le nombre de données est réduit et entraîne les incertitudes notables visibles plus haut. Mais cela ne remet pas en cause le but de ce travail qui rentre parfaitement dans le cadre du modèle établi par Nottale, Chaline et Grou ; nous avons souligné à plusieurs reprises les points communs aux études de ces auteurs et au travail présent. Et pour bien ancrer cette conviction nous avons entrepris de montrer la présence de la log-périodicité dans un grand nombre d'autres exemples.

Autre argument pour valoriser notre résultat : on doit noter que le temps critique Tc se situe au début du grand vide religieux, bien connu, qui s'étend de 1275/80 à 1350/60. À propos de cette époque, M. Pacaut [1993] peut écrire concernant les ordres monastiques :

La réalité, à l'aube du XIVe siècle, est donc celle du repli sur soi,de la stagnation, de l'atonie.

Plus loin, il confirme à propos des Franciscains et Dominicains :

Les Mendiants connaissent au cours des derniers siècles du Moyen Âge, sous l'effet des mêmes causes (crises économiques, troubles politiques et guerres, Grand Schisme) des difficultés aussi vives que celles que rencontrent les ordres monastiques et les instituts religieux plus anciens.

Soulignons aussi que c'est le moment où le pape Clément V s'installe à Avignon alors qu'une crise économique commence à frapper sévèrement l'Occident.

Les caractéristiques fractales des ordres que nous avons rappelées plus haut confirment que ceux-ci avaient été implantés de façon à optimiser leurs influences sur les populations, d'abord campagnardes, puis citadines. L'optimisation était limitée par des contraintes évidentes, d'où la fractalisation [Nottale, 1998]. Ces implantations étaient proches de celles du clergé séculier qui s'était installé auprès de ses églises dans chaque paroisse. Ces deux quadrillages des populations expliquent une partie de la concurrence entre les deux clergés au cours des siècles.

\section{CONCLUSION}

$\mathrm{Au}$ long de la période qui suit la grande crise des années 1300 , on ne peut que s'interroger sur ce que continuent à représenter la présence et l'action des Ordres à l'intérieur de l'Église catholique en Occident?

Jusqu'au terne XIVe siècle les ordres religieux et monastiques ont été les seuls corps puissants, innovants, constitués face aux pouvoirs laïques des rois, des seigneurs féodaux. Ce sont eux qui possédaient avec d'énormes bibliothèques la Culture, la Science, la Technique. Les Clunisiens, les Cisterciens ont pu bâtir des édifices remarquables dans des styles différents. Comme nous l'avons déjà écrit, ils ont fait progresser, en particulier les Cisterciens, les techniques de l'agriculture, de l'irrigation, de l'assolement, des moulins..., améliorations qui ont profité à leurs vastes domaines, mais, également, à toute la population environnante. Sur un plan politique, des religieux, comme saint Bernard en appelant à la croisade, ont eu une influence 
considérable sur l'Histoire. Plus tard, les Mendiants par leurs actions dans le domaine intellectuel, mais aussi artistique, par leurs enseignements ont influencé les sociétés plus fortement que le clergé séculier qui était, alors, au début de son organisation liée à celle de la société. Par contre, à partir du XVe siècle, le clergé séculier a commencé à jouer un rôle primordial dans les sociétés même si, sur le plan religieux, les réguliers ont participé, avec des succès, aux luttes de la Contre Réforme. Quand les difficultés dues à la Réforme sont apaisées, c'est un rapport inverse qui s'installe peu à peu. Les ordres vont avoir une marge d'actions plus restreinte, une grande partie de leurs efforts étant destinés à se reconstruire après les attaques qu'ils subissent et alors que leur indépendance est fortement limitée par les cardinaux du Vatican. Dorénavant, et cela jusqu'à aujourd'hui, ils se vouent assez exclusivement à de nombreuses et non négligeables tâches charitables et pédagogiques. Ce sont aussi d'efficaces propagandistes de la foi catholique et acteurs sociaux dans des pays non chrétiens d'Afrique, d'Amérique ou d'Asie.

La conclusion serait donc d'en déduire que le rôle historique, prépondérant des ordres religieux s'est situé entre le Ve et XIVe siècle. En reprenant le terme de «gain de productivité » défini par P.Grou [1987] dans ses études économiques comme facteur de développement, il est possible de dire que dans l'exemple étudié ici, les ordres majeurs se sont succédé au cours de cet intervalle de temps afin d'obtenir un gain de productivité maximum dans l'exercice de leurs activités religieuses. Quand ce gain faiblissait, il fallait qu'un ordre nouveau devienne prépondérant en apportant un idéal sans ombre et des pratiques nouvelles pour conduire l'Église. On peut proposer que, dans ce cadre, l'évolution des ordres a suivi une loi log-périodique définie plus haut. Puis, au cours des époques ultérieures, c'est l'Église séculaire qui a pris, en partie, la place. Nous retrouvons donc ici les mêmes démarches avec les mêmes causes et conséquences qu'avaient soulignées L. Nottale, J. Chaline et P. Grou dans leurs travaux portant sur des évolutions fort différentes en durées et en natures. Les événements extérieurs aux ordres (guerres, révolutions, peste, pillages, accidents climatiques...) qui ont accompagné les sauts de l'évolution (c'est-à-dire l'émergence d'ordres dominants nouveaux) se sont produits malheureusement tout au long des siècles en dehors de ces épisodes de ruptures et cela, sans entraîner les mêmes conséquences. Donc, ces péripéties ne sont pas les responsables principaux de ces sauts ; ils n'ont été déterminants que quand les ordres subissaient une certaine décadence.

La question finale reste toujours identique : pourquoi de telles universalités, quelles lois les pilotent ? Il est possible d'admettre que chaque ordre émergeant bénéficie de l'expérience des ordres prépondérants qui l'ont précédé, qu'il garde une certaine mémoire des processus antérieurs ce qui lui donne la possibilité de prendre des « raccourcis» dans son évolution et lui épargne du temps; mais pourquoi un même facteur d'auto similarité à chaque étape ?

Les trois auteurs précédents, dans les articles référencés plus haut, ont calculé qu'une super-crise devrait être attendue vers $2080+/$-30. Il serait intéressant de prolonger ce travail pour tenter de prévoir si, après cette date, les puissances économiques qui modèlent actuellement notre monde subiraient la même dilution, le même déclin (relatif, car il n'y a pas disparition !) que les ordres après le XIVe siècle ?

Remerciements. Nous souhaitons remercier L. Nottale (Observatoire Paris-Meudon), J. Chaline (Université de Bourgogne) et P. Grou (Université Versailles-Saint-Quentin) pour avoir, fort amicalement, relu et commenté ce texte. 


\section{BIBLIOGRAPHIE}

BERLIOZ J., HEITZ C., LE GOFF J., VOUCHEZ A., Moines et religieux au Moyen-Âge, Paris, Le Seuil, 1994.

BRISSAUD I., «La diffusion des ordres religieux et monastiques : un système critique auto-organisé ? », Bulletin de l'Union des Professeurs de Physique et de Chimie (BUP) 98, 2004, p. 137-148.

BRISSAUD I., «La chronologie du jazz suit-elle une loi log-périodique ? », Mathématiques et Sciences humaines 178, 2007, p. 41-50.

BRISSAUD I., BARON E., «La course des accélérateurs vers les hautes énergies et la log-périodicité », Revue Européenne de Géographie (Cybergeo) 15, décembre 2007.

CHALINE J., NOTTALE L., GROU P., «L'arbre de vie a t-il une structure fractale ? », Comptes rendus de l'Académie des Sciences 328, Paris, 1999, p. 717-726.

DUBOIS D., « Les ordres monastiques », Que sais-je ?, Presses Universitaires de France, 1998.

FRANKHAUSER P., La fractalité des structures urbaines, Paris, Anthropos,1994.

GROU P., L'aventure économique, Paris, L'Harmattan, 1987.

GROU P., NOTTALE L., CHALINE J., "Évolution morphologique et culturelle humaine : l'apport des modèles fractals", Colloque Événements rapides instabilités, changements culturels au quaternaire, 2002, p. 1-10.

JOHANSEN A. A., SORNETTE D., "Financial anti bubbles: log-periodicity in gold and Nikkei collapses", International Journal of Modern Physics C10, 1999, p. 563-575.

LE BRAS G., « Les ordres religieux », Paris, Flammarion, 1980.

LE GOFF J., REMOND R. (Dir.), Histoire de la France religieuse, Paris, Le Seuil, 1988.

LE GOFF J., Histoire de la France religieuse, Paris, Le Seuil, 1992.

LE GOFF J., La civilisation de l'Occident médiéval, Paris, Flammarion, 2000.

MARC-BONNET H., Histoire des ordres religieux, Paris, Presses Universitaires de France, 1968.

NOTTALE L., La relativité dans tous ses états, Paris, Hachette Littérature, 1998.

NOTTALE L, CHALINE J., GROU P., "On the fractal structure of evolutionary trees", Fractals in Biology and Medicine, 2000, p. 247-258.

NOTTALE L, CHALINE J., GROU P., Les arbres de l'évolution, Paris, Hachette Sciences, 2000.

PACAUT M., Les moines blancs, Paris, Fayard, 1993.

PACAUT M., Les ordres monastiques et religieux au Moyen-âge, Paris, Nathan, 1993.

SORNETTE D., JOHANSEN A.A., BOUCHAUD J.P., "Stock market crashes, precursors and replicas", Journal de Physique 6, 1996, p. 167-175.

SORNETTE D., "Discrete scale invariance and complex dimensions", Physics Reports 297, 1998, p. 239270. 